\title{
A Green Microwave-Assisted Synthesis of New Pyridazinium-Based Ionic Liquids as an Environmentally Friendly Alternative
}

\author{
Mouslim Messali", Saleh A. Ahmed \\ Chemistry Department, Faculty of Science, Taibah University, Al-Madina Al-Mounawara, Saudi Arabia \\ E-mail: ${ }^{*}$ mouslim@mail.be
}

Received May 24, 2011; revised June 29, 2011; accepted July 8, 2011

\begin{abstract}
A green microwave-assisted procedure for the preparation of a series of fifteen new $\mathrm{N}$-alkylpyridazinium ionic liquids with different functionality in the alkyl chain is described. For the first time, target ionic liquids were prepared using standard methodology and under microwave irradiation in short duration of time with quantitative yields. In most cases, the microwave-assisted reaction is an environmentally friendly alternative to traditional methods.
\end{abstract}

Keywords: Green Procedure, Pyridazinium-Salts, Ionic Liquids, Microwave Irradiation

\section{Introduction}

Over the past few decades the number of publications concerning room-temperature ionic liquids (RTILs) has increased substantially [1]. (RTILs) provide a new class of solvents where molecules are composed of ions. At normal temperatures, ionic liquids have essentially zerovapor pressure and are thermally stable over a wide range of temperature. Therefore, they are considered as environmentally friendly alternatives to the classical use of organic solvents.

RTILs have been also widely investigated for a variety of applications: the use as solvents or catalysts for chemical synthesis [2-3], media for electrodeposition of metals [4-6], electrolyte for electrochemical devices such as battery [7], supercapacitors [8,9], as inhibitors of corrosion and as fluids for thermal storage and exchange in solar concentrating power plants $[10,11]$.

Recently, several procedures have been recommended for Green Chemistry involving: solvent-free reactions [12], non-classical modes of activation such as ultrasounds or microwaves. The use of MW irradiation leads to large reductions in reaction times, enhancements in conversions, sometimes in selectivity, with several advantages of the eco-friendly approach [13-16]. The most common benefits of MW irradiation are: 1) very rapid reactions, frequently a few minutes, brought about by high and uniform temperatures and combined with pre- ssure effects, 2) higher degree of purity achieved due to short residence time at high temperatures, 3) yields often better, obtained within shorter times and with purer products [17].

\section{Results and Discussion}

In this work, we have synthesized a variety of a variety of new pyridazinium-based ionic liquids by microwave heating and the protocol is then compared with the similar preparation using conventional heating (oil bath at $80^{\circ} \mathrm{C}$ ). The comparative results are summarized in Table 1.

Initially, under standard conditions $\left(\mathrm{SC}_{1}\right.$ : toluene, $80^{\circ} \mathrm{C}$, $18 \mathrm{~h}$ ), the nucleophilic alkylation of pyridazine with different alkyl halides with different molar ratios (1:1.1; $1: 2$ and $1: 3 \mathrm{eq})$ afforded the corresponding monopyridiazinium halides in $78 \%-89 \%$ yield as solid powder after solvent removal by filtration (Scheme 1) [18]. Alternative anions were subsequently introduced by a metathesis reaction $\left(\mathrm{SC}_{2}\right.$ : Acetonitrile, $\left.70^{\circ} \mathrm{C}, 3 \mathrm{~h}\right)$, with slightly excess of anions namely, tetrafluoroborate or hexafluorophosphate (Scheme 1) [19]. The pure metathesis products were obtained after filtration of the salts (metal halides), then followed by filtrate evaporation and washing the residue with dichloromethane followed by further filtration to remove the excess of anion salts $\left(\mathrm{NaBF}_{4}, \mathrm{KPF}_{6}\right)$. Finally, evaporation of the filtrate afforded 
Table 1. Reaction conditions and reaction yields for the synthesis of pyridazinium-based ionic liquids using conventional preparation (SC) and under microwave irradiation (MW).

\begin{tabular}{ccccc}
\hline \multirow{2}{*}{ S.No. } & \multirow{2}{*}{$\mathrm{RX}$} & \multicolumn{3}{c}{ Yield (\%) } \\
& & $\mathrm{N}$-Alkylation (first step) & $\mathrm{Mp}\left({ }^{\circ} \mathrm{C}\right)$ \\
\cline { 3 - 4 } & & $\mathrm{SC}_{1}{ }^{\mathrm{a}}$ & $\mathrm{MW}^{\mathrm{b}}$ & \\
\hline 1 & $\mathrm{CH}_{3}\left(\mathrm{CH}_{2}\right)_{2} \mathrm{Br}$ & 82 & 95 & $104-106$ \\
2 & $\mathrm{CH}_{3}\left(\mathrm{CH}_{2}\right)_{3} \mathrm{Br}$ & 81 & 94 & $94-96$ \\
3 & $\mathrm{NC}\left(\mathrm{CH}_{2}\right)_{2} \mathrm{Br}$ & 87 & 94 & $90-91^{*}$ \\
4 & $\mathrm{NC}_{\left(\mathrm{CH}_{2}\right)_{3} \mathrm{Cl}}$ & 78 & 91 & $140-142$ \\
5 & $\mathrm{EtO}_{2} \mathrm{C}\left(\mathrm{CH}_{2}\right)_{3} \mathrm{Br}$ & 89 & 95 & $52-55$ \\
\hline
\end{tabular}

${ }^{a}$ Time $(18 \mathrm{~h})$, Temperature $\left(80^{\circ} \mathrm{C}\right.$ in Toluene; ${ }^{\mathrm{b}}$ Time $(20 \mathrm{~min})$, Temperature $\left(100^{\circ} \mathrm{C}\right)$, Power $(240 \mathrm{~W})$, Pressure $(40 \mathrm{psi}){ }^{*}$ hygroscopic material.

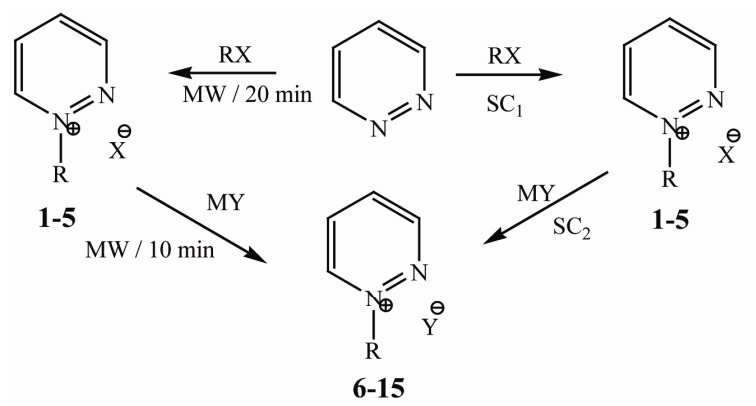

$\mathrm{R}=\mathrm{CH}_{3}\left(\mathrm{CH}_{2}\right)_{2}, \mathrm{CH}_{3}\left(\mathrm{CH}_{2}\right)_{3}, \mathrm{NC}\left(\mathrm{CH}_{2}\right)_{2}, \mathrm{NC}\left(\mathrm{CH}_{2}\right)_{3}, \mathrm{EtO}_{2} \mathrm{C}\left(\mathrm{CH}_{2}\right)_{3}$ $\mathrm{X}=\mathrm{Cl}, \mathrm{Br}$

$\mathrm{MY}=\mathrm{NaBF}_{4}, \mathrm{KPF}_{6}$

$\mathrm{SC}_{1}$ : toluene, $80^{\circ} \mathrm{C}, 18 \mathrm{~h} ; \mathrm{SC}_{2}$ : Acetonitrile, $70^{\circ} \mathrm{C}, 3 \mathrm{~h}$

Scheme 1. Synthesis of pyridazinium-based ionic liquids using standard conditions (SC) and under microwave irradiation (MW).

the desired ionic liquids in good yields.

It's noticeable that, the reactivity trend of halides is found to be in the order $\mathrm{Br}>\mathrm{Cl}$ as expected for an $\mathrm{S}_{\mathrm{N}} 2$ reaction, i.e. higher temperatures and longer reaction times are required with poorer leaving groups $\left(\mathrm{Cl}^{-}<\mathrm{Br}^{-}\right)$

On the other hand, the microwave-assisted preparation of pyridazinium-based ionic liquids, already synthesized by conventional methods, was explored further with the objective of shortening the reaction time. The reaction conditions were optimized by using either temperature or power control. Use of a small amount of solvent was necessary in these reactions, since the pyridaziniumhalides salts are solid. The optimum reaction conditions determined for the synthesis of these unknown ionic liquids was shown in Table 1.

Varma reported that an anion exchange metathesis is easily performed under MW activation using a domestic oven [20]. In this way, the preparation of pyridazinium tetrafluoroborate or hexafluorophosphate salts was carried out in the CEM Discover monomode system with a strict control of power and temperature during the reaction process. The data in Table 2 indicated that very good yields were obtained within very short reaction times. As observed, the anion nature of exchange agents did not affect the yields.

Interestingly, the alkylation of pyridazine with different molar rations of alkyl halides afforded only the mono alkylated pyridazium-salts by using both SC and MW methods without any evidence for formation of dialkylated products. These results were supported on the basis of NMR (see the spectroscopic supplementary materials) and molecular mechanics calculations (MM2). From the MM2 calculations, it's clear that the steric energies of the dialkaylated ionic liquids are higher than the monoalkylated products by factor ranged between 3-5 (Table 3, Figure 1). These high energies led to the instability of the dilakylated ionic liquid and prevent its formations. Indeed, the instability of the dialkylated ionic liquids can also be attributed to the electrostatic reputations between the two positively charges on the nitrogen atoms of the dialakylated products as well as the reputations of the two negatively charges of the anions outside the ring.

On the other hand, the UV spectra of selected synthesis ionic liquids (Figure 2) showed that, all synthesized ionic liquids have a colorless to pale brown color in water solution in concentration of $10^{-3} \mathrm{~mol} / 1$ solution as well as in solid state with very low absorbance in the visible region. A typical absorption in the UV region at $242-245 \mathrm{~nm}$ for selected synthesized ionic liquids with shift about $4 \mathrm{~nm}$ between them was detected. A broad bathchromic shift to visible region from 525 to $275 \mathrm{~nm}$ with low absorbance was monitored when the ionic liquid alkyl chain substituted with terminal chromophoric cyano group (Figure 2).

Table 2. Different entries, reaction conditions and reaction yields for the anion metathesis using conventional preparation (CP) and under microwave irradiation (MW).

\begin{tabular}{|c|c|c|c|c|c|c|}
\hline \multirow[t]{2}{*}{ S.No. } & \multirow[t]{2}{*}{$\mathrm{R}$} & \multirow[t]{2}{*}{$\mathrm{X}^{-}$} & \multirow[t]{2}{*}{$\mathrm{M} \mathrm{Y}^{+}$} & \multicolumn{2}{|c|}{$\begin{array}{c}\text { Yield (\%) } \\
\text { Anion } \\
\text { metathesis } \\
\text { (second step) }\end{array}$} & \multirow[t]{2}{*}{$\mathrm{Mp}\left({ }^{\circ} \mathrm{C}\right)$} \\
\hline & & & & $\mathrm{MW}^{\mathrm{a}}$ & $\mathrm{CP}^{\mathrm{b}}$ & \\
\hline 6 & $\mathrm{CH}_{3}\left(\mathrm{CH}_{2}\right)_{2}$ & $\mathrm{Br}^{-}$ & $\mathrm{NaBF}_{4}$ & 95 & 98 & Oil \\
\hline 7 & $\mathrm{CH}_{3}\left(\mathrm{CH}_{2}\right)_{2}$ & $\mathrm{Br}^{-}$ & $\mathrm{KPF}_{6}$ & 93 & 97 & Oil \\
\hline 8 & $\mathrm{CH}_{3}\left(\mathrm{CH}_{2}\right)_{3}$ & $\mathrm{Br}^{-}$ & $\mathrm{NaBF}_{4}$ & 94 & 98 & Oil \\
\hline 9 & $\mathrm{CH}_{3}\left(\mathrm{CH}_{2}\right)_{3}$ & $\mathrm{Br}^{-}$ & $\mathrm{KPF}_{6}$ & 94 & 98 & Oil \\
\hline 10 & $\mathrm{NC}\left(\mathrm{CH}_{2}\right)_{2}$ & $\mathrm{Br}^{-}$ & $\mathrm{NaBF}_{4}$ & 92 & 97 & $96-97$ \\
\hline 11 & $\mathrm{NC}\left(\mathrm{CH}_{2}\right)_{2}$ & $\mathrm{Br}^{-}$ & $\mathrm{KPF}_{6}$ & 93 & 98 & $102-103$ \\
\hline 12 & $\mathrm{NC}\left(\mathrm{CH}_{2}\right)_{3}$ & $\mathrm{Cl}^{-}$ & $\mathrm{NaBF}_{4}$ & 94 & 98 & Oil \\
\hline 13 & $\mathrm{NC}\left(\mathrm{CH}_{2}\right)_{3}$ & $\mathrm{Cl}^{-}$ & $\mathrm{KPF}_{6}$ & 93 & 97 & Oil \\
\hline 14 & $\mathrm{EtO}_{2} \mathrm{C}\left(\mathrm{CH}_{2}\right)_{3}$ & $\mathrm{Br}^{-}$ & $\mathrm{NaBF}_{4}$ & 92 & 98 & Oil \\
\hline 15 & $\mathrm{EtO}_{2} \mathrm{C}\left(\mathrm{CH}_{2}\right)_{3}$ & $\mathrm{Br}^{-}$ & $\mathrm{KPF}_{6}$ & 94 & 98 & Oil \\
\hline
\end{tabular}


Table 3. Steric and optimum energies of both mono- and dialkylakted pyridazine based ionic liquids.

\begin{tabular}{lcc}
\hline Alkyl halide & $\begin{array}{c}\text { Energy of } \\
\text { monoalkylation }\end{array}$ & $\begin{array}{c}\text { Energy of } \\
\text { dialkylation }\end{array}$ \\
\hline $\mathrm{CH}_{3}\left(\mathrm{CH}_{2}\right)_{2} \mathrm{Br}$ & -39.7923 & -120.1867 \\
$\mathrm{CH}_{3}\left(\mathrm{CH}_{2}\right)_{3} \mathrm{Br}$ & -39.3937 & -118.4058 \\
$\mathrm{NC}\left(\mathrm{CH}_{2}\right)_{2} \mathrm{Br}$ & -39.6971 & -105.8930 \\
$\mathrm{NC}\left(\mathrm{CH}_{2}\right)_{3} \mathrm{Cl}$ & -37.2709 & -113.8232 \\
$\mathrm{EtO}_{2} \mathrm{C}\left(\mathrm{CH}_{2}\right)_{3} \mathrm{Br}$ & -33.0301 & -165.4593 \\
\hline
\end{tabular}
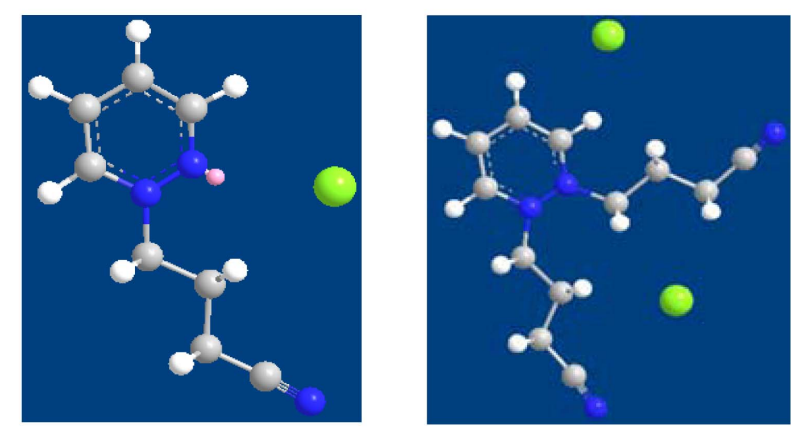

Figure 1. An example of the optimization of the energies using MM2 calculations of both mono- alkylated pyridazinium (4) and its corresponding dialkylated pyridazinium - based ionic liquids.

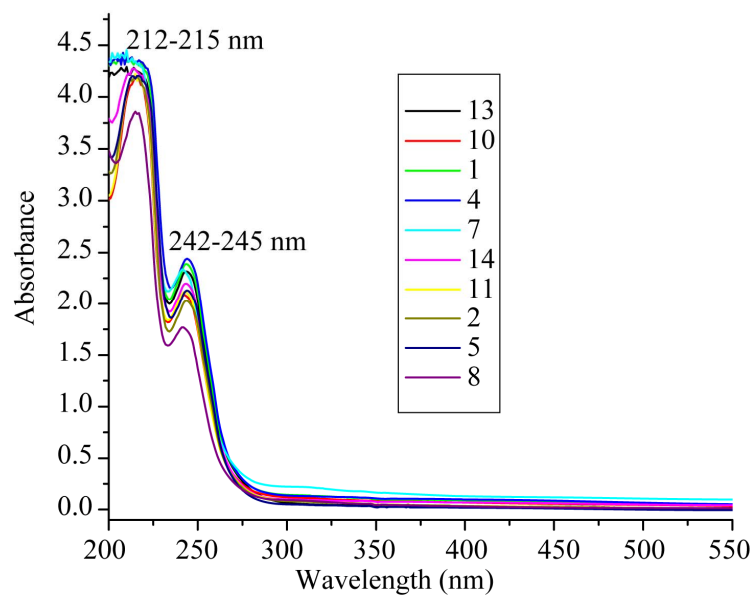

Figure 2. UV/VIS spectra of some selected ionic liquids ( $c=$ $\left.1 \times 10^{-3} \mathrm{~mol} / \mathrm{l}\right)$ in water solution.

\section{Conclusions}

In conclusion, new environmentally friendly pyridazinium-based ionic liquids were prepared by using microwave irradiation. Many advantages for the MW irradiation compared with the standard methods have been recorded. Increasing of the reaction molar ratios between pyridazine and the alkyl halides in both SC and MW did not show any evidence for formation of the dialkylated pyridazine-based ionic liquids. Comparison of SC and
MW methods afforded a lot of advantages and recommendations for the use of the green microwave assisted reactions. These synthesized ionic liquids will be used in the synthesis of novel photochromic materials based ionic liquids as a first attempts for preparation of water soluble photochromic materials based ionic liquid and will be discussed in details in the forthcoming paper.

\section{Experimental}

\subsection{General Comments}

All new compounds were synthesized and characterized by ${ }^{1} \mathrm{H}$ NMR, ${ }^{13} \mathrm{C}$ NMR, IR and LCMS.

${ }^{1} \mathrm{H}$ NMR $(400 \mathrm{MHz})$ and ${ }^{13} \mathrm{C}$ NMR $(100 \mathrm{MHz})$ spectra were measured in DMSO at room temperature at 400 MHz. Chemical shifts (d) were reported in ppm to a scale calibrated for tetramethylsilane (TMS), which is used as an internal standard. The LCMS spectra were measured with a Micromass, LCT mass spectrometer. IR spectra were recorded on a Schimadzu 8201 PC, FTIR spectrophotometer in $\mathrm{NaCl}$ and $\mathrm{KBr}$ discs $\left(\operatorname{vmax}\right.$ in $\left.\mathrm{cm}^{-1}\right)$. The microwave-assisted reactions were performed using a controllable single-mode microwave reactor, CEM Discovery, designed for synthetic use. The reactor is equipped with a magnetic stirrer as well as a pressure, temperature and power controls. The maximum operating pressure of the reactor is 20 bar. The power and temperature range are $15-300 \mathrm{~W}$ and $60^{\circ} \mathrm{C}-250^{\circ} \mathrm{C}$, respectively.

\subsection{General Procedures for the Synthesis of Pyridazinium Halides (1-5) using Conventional Method}

To the solution of pyridazine (1eq) in toluene, was added alkyl halide (1.1 eq) at room temperature, followed by stirring at $80^{\circ} \mathrm{C}$ for $18 \mathrm{~h}$. The completion of the reaction was marked by the separation of solid from the initially obtained clear and homogenous mixture of pyridazine and alkyl halide in toluene. The product was isolated by filtration to remove the unreacted starting materials and solvent. Subsequently, the quaternary salt was washed with ethyl acetate. In some cases, in each case, the IL/salt was finally dried at a reduced pressure to get rid of all the volatile organic compounds.

\subsection{General Procedure for the Synthesis of Pyridazinium Tetrafluoroborates or Hexafluorophosphates (6 - 15) using Conventional Method}

The quaternary salt (1 eq) was dissolved in acetonitrile to obtain a clear solution. To this solution of quaternary 
halide was added solution of sodium tetrafluoroborate or potassium hexafluorophosphate (1.2 eq), followed by stirring at $70^{\circ} \mathrm{C}$ for $3 \mathrm{~h}$. The product was isolated by solvent extraction using dichloromethane. The extracts were dried over anhydrous $\mathrm{MgSO}_{4}$ and evaporated under reduced pressure to yield the desired product.

\subsection{General Procedures for the Synthesis of Pyridazinium halides (1-5) under Microwave Irradiation}

Pyridazine (1 eq) and different alkyls halides (1 eq) were placed in a microwave reactor vessel and irradiated for 20 minutes at $100^{\circ} \mathrm{C}$. the product is collected as described in the conventional method.

\subsection{General Procedure for the Synthesis of Pyridazinium Tetrafluoroborate Hexafluorophosphates (6 - 15) under Microwave Irradiation}

Pyridazinium-halides salts ( $1 \mathrm{eq}$ ) were placed in a microwave reactor vessel, 1 eq of $\mathrm{NaBF}_{4}$ or $\mathrm{KPF}_{6}$ in a small amount of acetonitrile was added. The reaction was carried out for $10 \mathrm{~min}$ at $70^{\circ} \mathrm{C}$. the product is collected as described in the conventional method.

\section{Characterization}

\subsection{1-Propylpyridazin-1-ium Bromide (1)}

${ }^{1} \mathrm{H}$ NMR (400 MHz, DMSO) $\delta: 1.00(\mathrm{t}, J=7.6,3 \mathrm{H}), 2.16$ (quint, $J=7.6,2 \mathrm{H}), 4.86(\mathrm{t}, J=7.6,2 \mathrm{H}), 8.53-8.56(\mathrm{dd}$, $J=1.2,1 \mathrm{H}), \quad 8.61-8.65(\mathrm{dd}, J=1.2,1 \mathrm{H}), 9.53-9.54$ $(\mathrm{d}, J=1.21 \mathrm{H}), 9.73-9.74(\mathrm{~d}, J=1.21 \mathrm{H}) ;{ }^{13} \mathrm{C} \mathrm{NMR}$ (100MHz, DMSO) $\delta$ : $12.3\left(\mathrm{CH}_{3}\right), 25.8\left(\mathrm{CH}_{2}\right), 69.7\left(\mathrm{CH}_{2}\right)$, 138.4 (C-4), 139.0 (C-5), 151.7 (C-6), 157.1 (C-3).

\subsection{1-Butylpyridazin-1-ium Bromide (2)}

${ }^{1} \mathrm{H}$ NMR (400 MHz, DMSO) $\delta: 0.97(\mathrm{t}, J=7.2,3 \mathrm{H}), 1.42$ (quint, $J=7.2,2 \mathrm{H}), 2.10$ (quint, $J=7.2,2 \mathrm{H}), 4.90(\mathrm{t}, J=$ $7.2,2 \mathrm{H}), 8.55-8.56(\mathrm{dd}, J=1.2,1 \mathrm{H}), 8.61-8.63(\mathrm{dd}$, $J=1.2,1 \mathrm{H}), 9.53-9.54(\mathrm{~d}, J=1.21 \mathrm{H}), 9.74-9.76(\mathrm{~d}, J$ $=1.21 \mathrm{H}) ;{ }^{13} \mathrm{C} \mathrm{NMR}(100 \mathrm{MHz}, \mathrm{DMSO}) \delta: 15.2\left(\mathrm{CH}_{3}\right)$, $21.3\left(\mathrm{CH}_{2}\right), 34.1\left(\mathrm{CH}_{2}\right), 68.1\left(\mathrm{CH}_{2}\right), 138.4(\mathrm{C}-4), 139.0$ (C-5), 151.7 (C-6), 157.1 (C-3).

\subsection{1-(2-Cyanoethyl)Pyridazin-1-ium Bromide (3)}

${ }^{1} \mathrm{H}$ NMR (400 MHz, DMSO) $\delta: 3.46(\mathrm{t}, J=6,2 \mathrm{H}), 5.25(\mathrm{t}$, $J=6,2 \mathrm{H}), 8.62-8.66(\mathrm{dd}, J=1.2,1 \mathrm{H}), 8.69-8.73(\mathrm{dd}$, $J=1.2,1 \mathrm{H}), 9.61-9.63(\mathrm{~d}, J=1.21 \mathrm{H}), 9.82-9.84(\mathrm{~d}, J$
$=1.21 \mathrm{H}) ;{ }^{13} \mathrm{C}$ NMR (100MHz, DMSO) $\delta: 20.4\left(\mathrm{CH}_{2}\right)$, $62.5\left(\mathrm{CH}_{2}\right), 119.9(\mathrm{CN}), 138.7$ (C-4), 140.3 (C-5), 152.9 (C-6), 157.6 (C-3).

\subsection{1-(3-Cyanopropyl)Pyridazin-1-ium Chloride} (4)

${ }^{1} \mathrm{H}$ NMR (400 MHz, DMSO) $\delta: 2.48$ (quint, $J=7.2,2 \mathrm{H}$ ), $2.68(\mathrm{t}, J=7.2,2 \mathrm{H}), 4.99(\mathrm{t}, J=7.2,2 \mathrm{H}), 8.52-8.55(\mathrm{dd}$, $J=1.2,1 \mathrm{H}), 8.59-8.63(\mathrm{dd}, J=1.2,1 \mathrm{H}), 9.51-9.52(\mathrm{~d}$, $J=1.21 \mathrm{H}), 9.76-9.77(\mathrm{~d}, J=1.21 \mathrm{H}) ;{ }^{13} \mathrm{C}$ NMR $(100$ MHz, DMSO) $\delta$ : $13.9\left(\mathrm{CH}_{2}\right), 24.9\left(\mathrm{CH}_{2}\right), 63.8\left(\mathrm{CH}_{2}\right)$, 120.1 (CN), 136.1 (C-4), 137.2 (C-5), 150.0 (C-6), 154.8 (C-3).

\subsection{1-(4-Ethoxy-4-Oxobutyl)Pyridazin-1-ium Bromide (5)}

${ }^{1} \mathrm{H}$ NMR (400 MHz, DMSO) $\delta: 1.15$ (t, $\left.J=7.2,3 \mathrm{H}\right), 2.26$ (quint, $J=7.2,2 \mathrm{H}$ ), 2.50 (quint, $J=7.2,2 \mathrm{H}$ ), 4.03 (q, $J=$ $7.2,2 \mathrm{H}), 4.85(\mathrm{t}, J=7.2,2 \mathrm{H}), 8.55-8.56(\mathrm{dd}, J=1.2$, $1 \mathrm{H}), 8.61-8.63(\mathrm{dd}, J=1.2,1 \mathrm{H}), 9.53-9.54$ (d, $J=1.2$ $1 \mathrm{H}), 9.74-9.76(\mathrm{~d}, J=1.21 \mathrm{H}) ;{ }^{13} \mathrm{C} \mathrm{NMR}(100 \mathrm{MHz}$, DMSO) $\delta: 14.2\left(\mathrm{CH}_{3}\right), 24.9\left(\mathrm{CH}_{2}\right), 30.2\left(\mathrm{CH}_{2}\right), 60.4$ $\left(\mathrm{CH}_{2}\right), 64.1\left(\mathrm{CH}_{2}\right), 136.2(\mathrm{C}-4), 136.9(\mathrm{C}-5), 150.2(\mathrm{C}-6)$, 154.7 (C-3), 172.3 (CO).

\subsection{1-Propylpyridazin-1-ium Tetrafluoroborate} (6)

${ }^{1} \mathrm{H}$ NMR (400 MHz, DMSO) $\delta: 0.92(\mathrm{t}, J=7.6,3 \mathrm{H}), 2.04$ (quint, $J=7.6,2 \mathrm{H}), 4.77(\mathrm{t}, J=7.6,2 \mathrm{H}), 8.54-8.58(\mathrm{dd}$, $J=1.2,1 \mathrm{H}), 8.66-8.70(\mathrm{dd}, J=1.2,1 \mathrm{H}), 9.58-9.60(\mathrm{~d}$, $J=1.21 \mathrm{H}), 9.85-9.87(\mathrm{~d}, J=1.21 \mathrm{H}) ;{ }^{13} \mathrm{C} \mathrm{NMR}(100$ MHz, DMSO) $\delta$ : $10.3\left(\mathrm{CH}_{3}\right), 23.0\left(\mathrm{CH}_{2}\right), 66.2\left(\mathrm{CH}_{2}\right)$, 136.0 (C-4), 136.6 (C-5), 149.8 (C-6), 154.6 (C-3).

\subsection{1-Propylpyridazin-1-ium Hexafluorphosphate (7)}

${ }^{1} \mathrm{H}$ NMR (400 MHz, DMSO) $\delta: 0.94$ (t, $\left.J=7.6,3 \mathrm{H}\right), 2.05$ (quint, $J=7.6,2 \mathrm{H}), 4.79(\mathrm{t}, J=7.6,2 \mathrm{H}), 8.59-8.63$ (dd, $J=1.2,1 \mathrm{H}), 8.72-8.75(\mathrm{dd}, J=1.2,1 \mathrm{H}), 9.63-9.64(\mathrm{~d}$, $J=1.21 \mathrm{H}), 9.88-9.90(\mathrm{~d}, J=1.21 \mathrm{H}) ;{ }^{13} \mathrm{C}$ NMR $(100$ MHz, DMSO) $\delta$ : $10.2\left(\mathrm{CH}_{3}\right), 22.9\left(\mathrm{CH}_{2}\right), 65.9\left(\mathrm{CH}_{2}\right)$, 135.9 (C-4), 136.5 (C-5), 149.8 (C-6), 154.5 (C-3).

\subsection{1-Butylpyridazin-1-ium Tetrafluoroborate} (8)

${ }^{1} \mathrm{H}$ NMR (400 MHz, DMSO) $\delta: 0.92(\mathrm{t}, J=7.2,3 \mathrm{H}), 1.35$ (quint, $J=7.2,2 \mathrm{H}), 1.99$ (quint, $J=7.2,2 \mathrm{H}), 4.81(\mathrm{t}, J=$ $7.2,2 \mathrm{H}), 8.52-8.58(\mathrm{dd}, J=1.2,1 \mathrm{H}), 8.67-8.71(\mathrm{dd}, J$ 
$=1.2,1 \mathrm{H}), 9.59-9.61(\mathrm{~d}, J=1.21 \mathrm{H}), 9.88-9.89(\mathrm{~d}, J=$ $1.21 \mathrm{H}) ;{ }^{13} \mathrm{C}$ NMR $(100 \mathrm{MHz}, \mathrm{DMSO}) \delta: 13.3\left(\mathrm{CH}_{3}\right)$, $18.8\left(\mathrm{CH}_{2}\right), 34.4\left(\mathrm{CH}_{2}\right), 64.3\left(\mathrm{CH}_{2}\right), 136.0(\mathrm{C}-4), 136.6$ (C-5), 149.8 (C-6), 154.5 (C-3).

\subsection{1-Butylpyridazin-1-ium Hexafluorphosphate} (9)

${ }^{1} \mathrm{H}$ NMR (400MHz, DMSO) $\delta: 0.93$ (t, $\left.J=7.2,3 \mathrm{H}\right), 1.34$ (quint, $J=7.2,2 \mathrm{H}$ ), 1.99 (quint, $J=7.2,2 \mathrm{H}), 4.81$ (t, $J=$ $7.2,2 \mathrm{H}), \quad 8.56-8.60(\mathrm{dd}, J=1.2,1 \mathrm{H}), \quad 8.70-8.72(\mathrm{dd}, J$ $=1.2,1 \mathrm{H}), 9.60-9.61(\mathrm{~d}, J=1.21 \mathrm{H}), 9.90-9.92(\mathrm{~d}, J=$ $1.21 \mathrm{H}) ;{ }^{13} \mathrm{C}$ NMR $(100 \mathrm{MHz}, \mathrm{DMSO}) \delta: 13.2\left(\mathrm{CH}_{3}\right)$, $18.7\left(\mathrm{CH}_{2}\right), 31.4\left(\mathrm{CH}_{2}\right), 64.4\left(\mathrm{CH}_{2}\right), 135.9(\mathrm{C}-4), 136.5$ (C-5), 149.8 (C-6), 154.5 (C-3).

\subsection{1-(2-Cyanoethyl)Pyridazin-1-ium Tetrafluoroborate (10)}

${ }^{1} \mathrm{H}$ NMR (400 MHz, DMSO) $\delta: 3.37$ (t, $J=6,2 \mathrm{H}$ ), 5.13 (t, $J=6,2 \mathrm{H}), 8.64-8.68(\mathrm{dd}, J=1.2,1 \mathrm{H}), 8.77-8.80$ (dd, $J=1.2,1 \mathrm{H}), 9.67-9.68$ (d, $J=1.21 \mathrm{H}), 9.88-9.89$ $(\mathrm{d}, J=1.21 \mathrm{H}) ;{ }^{13} \mathrm{C}$ NMR (100MHz, DMSO) $\delta: 17.7$ $\left(\mathrm{CH}_{2}\right), 59.3\left(\mathrm{CH}_{2}\right), 117.5(\mathrm{CN}), 136.1(\mathrm{C}-4), 137.4(\mathrm{C}-5)$, 150.8 (C-6), 154.6 (C-3).

\subsection{1-(2-Cyanoethyl)Pyridazin-1-ium Hexafluorphosphate (11)}

${ }^{1} \mathrm{H}$ NMR (400 MHz, DMSO) $\delta: 3.38$ (t, $\left.J=6,2 \mathrm{H}\right), 5.13$ (t, $J=6,2 \mathrm{H}), 8.65-8.68$ (dd, $J=1.2,1 \mathrm{H}), 8.77-8.81$ (dd, $J=1.2,1 \mathrm{H}), 9.68-9.69$ (d, $J=1.21 \mathrm{H}), 9.89-9.90$ $(\mathrm{d}, J=1.21 \mathrm{H}) ;{ }^{13} \mathrm{C}$ NMR $(100 \mathrm{MHz}$, DMSO) $\delta: 17.7$ $\left(\mathrm{CH}_{2}\right), 59.3\left(\mathrm{CH}_{2}\right), 117.5(\mathrm{CN}), 136.0(\mathrm{C}-4), 137.4(\mathrm{C}-5)$, 150.8 (C-6), 154.6 (C-3).

\subsection{1-(3-Cyanopropyl)Pyridazin-1-ium Tetrafluoroborate (12)}

${ }^{1} \mathrm{H}$ NMR (400 MHz, DMSO) $\delta: 2.36$ (quint, $J=7.2,2 \mathrm{H}$ ), $2.68(\mathrm{t}, J=7.2,2 \mathrm{H}), 4.88(\mathrm{t}, J=7.2,2 \mathrm{H}), 8.59-8.62(\mathrm{dd}$, $J=1.2,1 \mathrm{H}), 8.71-8.74(\mathrm{dd}, J=1.2,1 \mathrm{H}), 9.61-9.62(\mathrm{~d}$, $J=1.21 \mathrm{H}), 9.86-9.87(\mathrm{~d}, J=1.21 \mathrm{H}) ;{ }^{13} \mathrm{C}$ NMR $(100$ $\mathrm{MHz}, \mathrm{DMSO}) \delta: 13.6\left(\mathrm{CH}_{2}\right), 25.1\left(\mathrm{CH}_{2}\right), 63.2\left(\mathrm{CH}_{2}\right)$, 119.7 (CN), 136.1 (C-4), 136.9 (C-5), 150.5 (C-6), 154.5 (C-3).

\subsection{1-(3-Cyanopropyl)Pyridazin-1-ium Hexafluorphosphate (13)}

${ }^{1} \mathrm{H}$ NMR (400 MHz, DMSO) $\delta: 2.34$ (quint, $J=7.2,2 \mathrm{H}$ ), $2.69(\mathrm{t}, J=7.2,2 \mathrm{H}), 4.91(\mathrm{t}, J=7.2,2 \mathrm{H}), 8.61-8.64(\mathrm{dd}$, $J=1.2,1 \mathrm{H}), 8.73-8.77$ (dd, $J=1.2,1 \mathrm{H}), 9.64-9.65$ (d,
$J=1.21 \mathrm{H}), 10.0-10.1(\mathrm{~d}, J=1.21 \mathrm{H}) ;{ }^{13} \mathrm{C} \mathrm{NMR}(100$ $\mathrm{MHz}, \mathrm{DMSO}) \delta: 13.5\left(\mathrm{CH}_{2}\right), 25.1\left(\mathrm{CH}_{2}\right), 63.1\left(\mathrm{CH}_{2}\right)$, $119.6(\mathrm{CN}), 136.0(\mathrm{C}-4), 136.9$ (C-5), 150.5 (C-6), 154.5 (C-3).

\subsection{1-(4-Ethoxy-4-Oxobutyl)Pyridazin-1-ium Bromide (14)}

${ }^{1} \mathrm{H}$ NMR (400MHz, DMSO) $\delta: 1.26$ (t, $\left.J=7.2,3 \mathrm{H}\right), 2.44$ (quint, $J=7.2,2 \mathrm{H}$ ), 2.61 (quint, $J=7.2,2 \mathrm{H}$ ), 4.17 (q, $J=$ $7.2,2 \mathrm{H}), 4.97$ (t, $J=7.2,2 \mathrm{H}), 8.54-8.58$ (dd, $J=1.2$, $1 \mathrm{H}), 8.62-8.65(\mathrm{dd}, J=1.2,1 \mathrm{H}), 9.54-9.55(\mathrm{~d}, J=1.2$ 1H), $9.75-9.77(\mathrm{~d}, J=1.21 \mathrm{H}) ;{ }^{13} \mathrm{C}$ NMR $(100 \mathrm{MHz}$, DMSO) $\delta: 15.8\left(\mathrm{CH}_{3}\right), 27.1\left(\mathrm{CH}_{2}\right), 32.9\left(\mathrm{CH}_{2}\right), 64.5$ $\left(\mathrm{CH}_{2}\right), 67.2\left(\mathrm{CH}_{2}\right), 138.5(\mathrm{C}-4), 139.3(\mathrm{C}-5), 152.1(\mathrm{C}-6)$, $157.2(\mathrm{C}-3), 177.3(\mathrm{CO})$.

\subsection{1-(4-Ethoxy-4-Oxobutyl)Pyridazin-1-ium Bromide (15)}

${ }^{1} \mathrm{H}$ NMR (400 MHz, DMSO) $\delta: 1.17(\mathrm{t}, J=7.2,3 \mathrm{H})$, 2.29 (quint, $J=7.2,2 \mathrm{H}$ ), 2.49 (quint, $J=7.2,2 \mathrm{H}$ ), 4.05 (q, $J=7.2,2 \mathrm{H}), 4.85$ (t, $J=7.2,2 \mathrm{H}), 8.57-8.60$ (dd, $J=$ $1.2,1 \mathrm{H}), 8.69-8.72(\mathrm{dd}, J=1.2,1 \mathrm{H}), 9.60-9.62(\mathrm{~d}, J=$ $1.21 \mathrm{H}), 9.89-9.91(\mathrm{~d}, J=1.21 \mathrm{H}) ;{ }^{13} \mathrm{C}$ NMR $(100 \mathrm{MHz}$, DMSO) $\delta$ : $14.0\left(\mathrm{CH}_{3}\right), 24.7\left(\mathrm{CH}_{2}\right), 30.0\left(\mathrm{CH}_{2}\right), 60.1$ $\left(\mathrm{CH}_{2}\right), 63.9\left(\mathrm{CH}_{2}\right), 136.0(\mathrm{C}-4), 136.7$ (C-5), 150.2 (C-6), 154.5 (C-3), 172.0 (CO).

\section{Acknowledgements}

The authors gratefully acknowledge the financial support from Taibah University (Grant 430/417) and thanks Dr Ziad Moussa for his assistance on the NMR analysis and fruitful discussion.

\section{Supplementary Data}

Supplementary data $\left({ }^{1} \mathrm{H}\right.$ NMR, ${ }^{13} \mathrm{C}$ NMR, ${ }^{11} \mathrm{~B}$ NMR, ${ }^{19} \mathrm{~F}$ NMR and ${ }^{31} \mathrm{P}$ NMR spectra) associated with this article are available.

\section{References}

[1] R. D. Rogers and K. Seddon, "Ionic Liquids: Industrial Applications for Green Chemistry," Oxford University Press, Oxford, 2002.

[2] J. F. Liu, G. B. Jiang, Y. G. Chi, Y. Q. Cai, Q. X. Zhou and J. T. Hu, "Use of Ionic Liquids for Liquid-Phase Microextraction of Polycyclic Aromatic Hydrocarbons," Analytical Chemistry, Vol. 75, No. 21, 2003, pp. 58705876. doi:10.1021/ac034506m

[3] J. H. Wang, D. H. Cheng, X. Y. Chen, Z. Du and Z. L. Fang, "Direct Extraction of Double-Stranded DNA into 
Ionic Liquid 1-Butyl-3-Methylimidazolium Hexafluorophosphate and Its Quantification," Analytical Chemistry, Vol. 79, No. 2, 2007, 620. doi:10.1021/ac061145c

[4] F. Endres, "Ionic Liquids, Solvents for the Electro-deposition of Metals and Semiconductors," ChemPhysChem, Vol. 3, No. 2, 2002, pp. 144-154.

doi:10.1002/1439-7641(20020215)3:2<144::AID-CPHC1 44>3.0.CO;2-\#

[5] Y. F. Lin and S. I. Wen, "Electrodeposition of Zinc from a Lewis Acidic Zinc Chloride-1-Ethyl-3-Methylimidazolium Chloride Molten Salt," Electrochimica Acta, Vol. 44, No. 16, 1999, pp. 2771-2777. doi:10.1016/S0013-4686(99)00003-1

[6] S. Takahashi, N. Koura, S. Kohara, M. L. Saboungi and L. A. Curtiss, "Technological and Scientific Issues of RoomTemperature Molten Salts," Plasmas Ions, Vol. 2, No. 34, 1999, pp. 91-105. doi:10.1016/S1288-3255(99)00105-7

[7] J. F. Brennecke and E. J. Magin, "Ionic Liquids: Innovative Fluids for Chemical Processing," AIChE Journal, Vol. 47, No. 11, 2001, pp. 2384-2389. doi:10.1002/aic.690471102

[8] M. Ue, M. Takeda, A. Toriumi, A. Kominato, R. Hagiwara and Y. Ito, "Application of Low-Viscosity Ionic Liquid to the Electrolyte of Double-Layer Capacitors," Journal of the Electrochemical Society, Vol. 150, No. 4, 2003, pp. A499-A502. doi:10.1149/1.1559069

[9] A. Balducci, U. Bardi, S. Caporali, M. Mastragostino and F. Soavi, "Ionic Liquids for Hybrid Supercapacitors," Electrochemistry Communications, Vol. 6, No. 6, 2004, pp. 566-570. doi:10.1016/j.elecom.2004.04.005

[10] M. Messali, “A Green Microwave-Assisted Synthesis, Characterization and Comparative Study of New Pyridazinium-Based Ionic Liquids Derivatives towards Corrosion of Mild Steel in Acidic Environment," Journal of Materials and Environmental Science, Vol. 2, No. 2, 2011, pp. 174-185.

[11] L. Moens, D. M. Blake, D. L. Rudnicki and M. J. Hale, "Advanced Thermal Storage Fluids for Solar Parabolic Trough Systems," Journal of Solar Energy Engineering, Vol. 125, No. 1, 2003, pp. 112-116. doi:10.1115/1.1531644
[12] P. T. Anastas and J. C.Warner, "Green Chemistry, Theory and Practice," Oxford University Press, Oxford, UK, 1998.

[13] A. Loupy, "Solvent-Free Microwave Organic Synthesis as an Efficient Procedure for Green Chemistry," Comptes Rendus Chimie, Vol. 7, No. 2, 2004, pp. 103-112.

[14] A. Aupoix, B. Pegot and G. Vo-Thanh, "Synthesis of Imidazolium and Pyridinium-Based Ionic Liquids and Application of 1-Alkyl-3-Methylimidazolium Salts as Pre-catalysts for the Benzoin Condensation Using Solvent-Free and Microwave Activation," Tetrahedron, Vol. 66, No. 6, 2010, pp. 1352-1356. doi:10.1016/j.tet.2009.11.110

[15] F. Yi, Y. Peng and G. Song, "Microwave-Assisted Liquid-Phase Synthesis of Methyl 6-Amino-5-Cyano-4-Aryl2-Methyl-4H-Pyran-3-Carboxylate Using Functional Ionic Liquid as Soluble Support," Tetrahedron Letters, Vol. 46, No. 22, 2005, pp. 3931-3933. doi:10.1016/j.tetlet.2005.03.197

[16] V. Singh, S. Kaur, V. Sapehiyia, J. Singh and G. L. Kad, "Microwave Accelerated Preparation of $[\mathrm{Bmim}]\left[\mathrm{HSO}_{4}\right]$ Ionic Liquid: An Acid Catalyst for Improved Synthesis of Coumarins," Catalysis Communications, Vol. 6, No. 1, 2005, pp. 57-60. doi:10.1016/j.catcom.2004.10.011

[17] M. Deetlefs and K. R. Seddon, "Improved Preparations of Ionic Liquids Using Microwave Irradiation," Green Chemistry, Vol. 5, 2003, No. 2, pp. 181-186. doi: $10.1039 / \mathrm{b} 300071 \mathrm{k}$

[18] M. Messali, "A Facile and Green Microwave-Assisted Synthesis of New Functionalized Picolinium-Based Ionic Liquids," Arabian Journal of Chemistry, In Press, Available Online, July 2011. doi:10.1016/j.arabjc.2011.06.030

[19] J. G. Huddleston, A. E. Visser, W. M. Reichert, H. D. Willauer, G. A. Broker and R. D. Rogers, "Characterization and Comparison of Hydrophilic and Hydrophobic Room Temperature Ionic Liquids Incorporating the Imidazolium Cation," Green Chemistry, Vol. 3, No. 4, 2001, pp. 156-164. doi:10.1039/b103275p

[20] R. S. Varma and V. V. Namboodiri, "Solvent-Free Preparation of Ionic Liquids Using a Household Microwave Oven," Pure and Applied Chemistry, Vol. 73, No. 8, 2001, pp. 1309-1313. doi: $10.1351 / \mathrm{pac} 200173081309$ 